\title{
Persistence of Coron's solution in nearly critical problems
}

\author{
Monica Musso And Angela Pistoia
}

\begin{abstract}
We consider the problem

$$
\begin{cases}-\Delta u=u^{\frac{N+2}{N-2}+\lambda} & \text { in } \Omega \backslash \varepsilon \omega, \\ u>0 & \text { in } \Omega \backslash \varepsilon \omega, \\ u=0 & \text { on } \partial(\Omega \backslash \varepsilon \omega),\end{cases}
$$

where $\Omega$ and $\omega$ are smooth bounded domains in $\mathbb{R}^{N}, N \geq 3, \varepsilon>0$ and $\lambda \in \mathbb{R}$. We prove that if the size of the hole $\varepsilon$ goes to zero and if, simultaneously, the parameter $\lambda$ goes to zero at the appropriate rate, then the problem has a solution which blows up at the origin.
\end{abstract}

Mathematics Subject Classification (2000):35J60 (primary); 35J25 (secondary).

\section{Introduction}

In this paper we are interested in the following problem

$$
\begin{cases}-\Delta u=u^{\frac{N+2}{N-2}+\lambda} & \text { in } D, \\ u>0 & \text { in } D, \\ u=0 & \text { on } \partial D,\end{cases}
$$

where $D$ is a smooth bounded domain in $\mathbb{R}^{N}, N \geq 3$ and $\lambda$ is a real parameter. The exponent $\frac{N+2}{N-2}$ is the so called critical Sobolev exponent for the Sobolev embedding $H_{0}^{1}(\Omega) \hookrightarrow L^{\frac{2 N}{N-2}}(\Omega)$.

It is well known that if $\lambda<0$, namely in the sub-critical regime, problem (1.1) has at least one solution for any domain $D$. In the last decades, several results on multiplicity and qualitative behavior of solutions to (1.1) in the slightly subcritical regime, namely when $\lambda \rightarrow 0^{-}$, have been obtained. We refer the readers for example to $[4,15,23,25]$.

When $\lambda=0$ or when $\lambda>0$ solvability of (1.1) is a much more delicate issue and depends strongly on the geometry of the domain $D$. Indeed if $\lambda \geq 0$ and $D$ is starshaped, then Pohozaev's identity [22] shows that problem (1.1) has no solution.

The authors are supported by the M.I.U.R. National Project "Metodi variazionali e topologici nello studio di fenomeni non lineari". The first author is supported by Fondecyt 1040936 (Chile). Received December 14, 2006; accepted in revised form May 23, 2007. 
As far as it concerns existence of solutions to (1.1) when $\lambda \geq 0$ we mention the following results. Assume first $\lambda=0$. Kazdan and Warner showed in [16] that if $D$ is an annulus then (1.1) has a solution, which is radially symmetric. Successively, Coron in [8] proved the existence of a solution to (1.1) provided $D$ has a small and not necessarily symmetric hole. Substantial improvement of this result was obtained by Bahri and Coron in [3] (see also [2]), showing that if some homology group of $D$ with coefficients in $Z_{2}$ is not trivial then problem (1.1) has at least one solution.

Going back to Coron's result, an interesting issue is the study of the asymptotic behavior of Coron's solution as the size of the hole tends to zero. In the case in which the hole is a ball of radius $r$ then the solution found by Coron concentrates around the hole and it converges, as $r \rightarrow 0$, in the sense of measure to a Dirac delta centered at the center of the hole. In the literature this is what is known as a solution with a (simple) bubble, at the center of the hole. We refer the reader to $[18,19,24]$ where the study of existence of positive solutions to (1.1) in domains with several small circular holes and their asymptotic behaviour as the size of the holes goes to zero is carried out.

Assume now that $\lambda>0$ and small enough. Del Pino, Felmer and Musso in [12] prove existence of a solution to (1.1) when $D$ has a small but not necessarily symmetric hole; they show that such a solution develops, as $\lambda \rightarrow 0^{+}$, two different bubbles, which, unlikely the one found by Coron, are centered in points which belong to the inside of $D$, even though they are extremely close to the hole. Successively and in the same setting treated in [12], Pistoia and Rey in [21] prove existence of a solution to (1.1), with three different spikes as the parameter $\lambda \rightarrow 0^{+}$. Under the assumption that $D$ is also symmetric, existence of solutions to (1.1), with an arbitrary number of bubbles as $\lambda \rightarrow 0^{+}$, has been proved in [13,20,21]. Let us finally remark that, in contrast with the sub-critical regime, there is no chance to find a solution to problem (1.1) exihibiting just one bubble inside the domain as $\lambda \rightarrow 0^{+}$as proven in [5].

It is interesting to point out that all the solutions found in the previously quoted papers for both the slightly super-critical and sub-critical regime, do not correspond in the limit as $\lambda \rightarrow 0^{ \pm}$to the one found by Coron, since they all disappear as $\lambda \rightarrow 0^{ \pm}$. Dancer conjectured in [9-11] that Coron's solution still exists for small $\lambda$. The aim of the present paper is precisely to show the validity of Dancer's conjecture, namely the persistence of Coron's solution for small $\lambda$, not necessarily positive.

Indeed, we prove that the solution found by Coron persists if we take $\lambda \neq 0$ and small and the size of the hole tends to zero, provided some link holds between the rate at which $\lambda$ goes to zero and the size of the hole. More precisely, we consider the problem

$$
\begin{cases}-\Delta u=u^{p+\lambda} & \text { in } \Omega \backslash \varepsilon \omega, \\ u>0 & \text { in } \Omega \backslash \varepsilon \omega, \\ u=0 & \text { on } \partial(\Omega \backslash \varepsilon \omega),\end{cases}
$$


where $\varepsilon>0, p=\frac{N+2}{N-2}, N \geq 3$ and $\lambda$ is a real parameter. Here $\omega$ is a smooth bounded domain in $\mathbb{R}^{N}$, not necessarily symmetric, such that $B\left(0, R_{1}\right) \subset \omega \subset$ $B\left(0, R_{2}\right)$, for some fixed number $0<R_{1}<R_{2}$. Hence $\varepsilon \omega$ represents the small but not necessarily symmetric hole and, as $\varepsilon \rightarrow 0$ the small hole shrinks to a point, namely the origin. $\Omega$ is a smooth bounded domain in $\mathbb{R}^{N}$ which contains the origin. Our result is the following.

Theorem 1.1. Let $\Lambda \in \mathbb{R}$ be fixed. There exists $\varepsilon_{0}>0$ such that for any $\varepsilon \in\left(0, \varepsilon_{0}\right)$ and for any $\Lambda_{\varepsilon} \in \mathbb{R}$ with $\lim _{\varepsilon \rightarrow 0} \Lambda_{\varepsilon}=\Lambda$, there exists a solution $u_{\varepsilon}$ to problem (1.2) with $\lambda=\Lambda_{\varepsilon} \varepsilon^{\frac{N-2}{2}}$, such that

$$
\left|\nabla u_{\varepsilon}\right|^{2} d x \rightarrow C_{N} \delta_{0} \text { in the sense of measures, as } \varepsilon \rightarrow 0,
$$

where $C_{N}=\alpha_{N}^{p+1} \int_{\mathbb{R}^{N}}\left(1+|y|^{2}\right)^{-(N+2) / 2} d y$.

Let us mention that the center of the bubble developed, as $\varepsilon \rightarrow 0$, by the solution found in the previous Theorem is at the center of the dropped hole. Thus the point where the solution concentrates does not belong to the domain. Taking into account that around the hole the solution concentrates but at the same time it has to satisfy zero Dirichlet boundary conditions, it is quite delicate to study the behavior of the solution in the region around the hole. An important estimate is contained in the crucial Lemma 2.2 in Section 2.

The proof of the result relies on a well known Ljapunov-Schmidt reduction. In Section 3 we describe the scheme of the proof that leads to Theorem 1.1. We leave to Sections 5 and 6 the detailed proofs of the results contained here. Section 4 is devoted to the delicate expansion of the energy functional associated to the problem evaluated at the ansatz for the solution.

\section{Ansatz and estimate of the first approximation}

In this section we describe a first approximation of the solution whose existence and properties are stated in Theorem 1.1.

Let $\mu$ be a positive real number and $\xi$ be a point in $\mathbb{R}^{N}$. The basic element to construct a solution to problem (1.2) is the function $U_{\mu, \xi}$ defined by

$$
U_{\mu, \xi}(x)=\alpha_{N} \frac{\mu^{\frac{N-2}{2}}}{\left(\mu^{2}+|x-\xi|^{2}\right)^{\frac{N-2}{2}}}, \mu>0, \xi \in \mathbb{R}^{N},
$$

with $\alpha_{N}:=[N(N-2)]^{\frac{N-2}{4}}$. It is well known (see $\left.[1,7,26]\right)$ that these functions are the solutions of the equation $-\Delta u=u^{p}$ in $\mathbb{R}^{N}$. Problem (1.2) is defined on a bounded domain and its solutions must satisfy zero Dirichlet boundary conditions. 
For this reason, we modify $U_{\mu, \xi}$ with its projection onto $H_{0}^{1}(\Omega \backslash \varepsilon \omega)$, namely we denote by $P_{\varepsilon} U_{\mu, \xi}$ the solution to the problem

$$
\begin{cases}-\Delta P_{\varepsilon} U_{\mu, \xi}=U_{\mu, \xi}^{p} & \text { in } \Omega \backslash \varepsilon \omega \\ P_{\varepsilon} U_{\mu, \xi}=0 & \text { on }(\partial \Omega \backslash \varepsilon \omega) .\end{cases}
$$

Let $\sigma:=\frac{N-2}{4}$. We assume that

$$
\mu:=d \sqrt{\varepsilon}, \delta<d<\delta^{-1} \text { and } \xi:=\mu \varepsilon^{\sigma} \tau, \tau \in \mathbb{R}^{N},|\tau|<\delta^{-1},
$$

for some positive and small fixed $\delta$. Moreover, we assume that the exponent $p+\lambda$ in (1.2) depends on $\varepsilon$, more precisely we will make the following choice for $\lambda$

$$
\lambda:=\Lambda_{\varepsilon} \varepsilon^{\frac{N-2}{2}}, \text { where } \lim _{\varepsilon \rightarrow 0} \Lambda_{\varepsilon}=\Lambda \in \mathbb{R} .
$$

We will look for a solution to (1.2) of the form $u=P_{\varepsilon} U_{\mu, \xi}+\psi$, where $P_{\varepsilon} U_{\mu, \xi}$ represents the leading term and $\psi$ is just a lower order term.

The main purpose of what remains of this section is the careful description of the asymptotic behavior of the projection $P_{\varepsilon} U_{\mu, \xi}(x)$, for $x \in \Omega \backslash \varepsilon \omega$. Observe that the function $U_{\mu, \xi}(x)$ attains its maximum value of order $\mu^{-\frac{N-2}{2}}$ at the point $\xi$. The choice (2.1) for the point $\xi$ implies that $\xi$ is located very much inside the hole $\varepsilon \omega$, as far as $\varepsilon$ is small and $N \geq 4$. For this reason, the correction to perform on $U_{\mu, \xi}(x)$ in order to satisfy zero boundary condition has to be huge in the region around the hole. This is the content of Lemma 2.2. In order to perform the expansion contained in Lemma 2.2, a technical difficulty is the fact that the hole $\omega$ is neither a ball nor a symmetric domain. To overcome this difficulty, we consider the problem

$$
\begin{cases}-\Delta u=0 & \text { in } \mathbb{R}^{N} \backslash \omega, \\ u=1 & \text { on } \partial \omega, \\ u \in \mathcal{D}^{1,2}\left(\mathbb{R}^{N} \backslash \omega\right) . & \end{cases}
$$

The following result holds.

Lemma 2.1. Problem (2.3) has a unique solution $\varphi_{\omega}$. Moreover,

$$
\frac{c_{1}}{|x|^{N-2}} \leq \varphi_{\omega}(x) \leq \frac{c_{2}}{|x|^{N-2}} \quad \forall x \in \mathbb{R}^{N} \backslash \omega
$$

for some positive constants $c_{1}, c_{2}$. Furthermore,

$$
\lim _{|x| \rightarrow+\infty}|x|^{N-2} \varphi_{\omega}(x)=c_{\omega}
$$

with

$$
c_{\omega}=\frac{1}{(N-2)\left|S^{N-1}\right|} \int_{\mathbb{R}^{N} \backslash \omega}\left|\nabla \varphi_{\omega}(x)\right|^{2} d x .
$$


Proof. We apply the Kelvin transform $\mathcal{T}$. Let $v(y):=|y|^{2-N} u\left(\frac{y}{|y|^{2}}\right), y \in \mathcal{T}(\omega)$. Then, $u$ solves (2.3) if and only if $v$ solves

$$
\begin{cases}-\Delta v=0 & \text { in } \mathcal{T}(\omega) \\ v=|y|^{2-N} & \text { on } \partial \mathcal{T}(\omega) \\ v \in \mathrm{H}^{1}(\mathcal{T}(\omega)) & \end{cases}
$$

Since $0 \notin \partial \mathcal{T}(\omega)$, problem (2.4) has a unique solution which satisfies $c_{1} \leq v(y) \leq$ $c_{2}$ for any $y \in \mathcal{T}(\omega)$ and $\lim _{y \rightarrow 0} v(y)=v(0)$. Therefore, the claim follows taking $c_{\omega}:=v(0)$.

We remark that if $\omega$ is the unit ball $B(0,1)$, then the function $\varphi_{\omega}$ is simply given by $\varphi_{\omega}(x)=\frac{1}{|x|^{N-2}}$.

Lemma 2.2. Let $\delta>0$ be fixed. If (2.1) holds true, then the following fact holds true

$$
P_{\varepsilon} U_{\mu, \xi}(x)=U_{\mu, \xi}(x)-\alpha_{N} \mu^{\frac{N-2}{2}} H(x, \xi)-\alpha_{N}\left(\frac{1}{\mu}\right)^{\frac{N-2}{2}} \varphi_{\omega}\left(\frac{x}{\varepsilon}\right)+R_{\varepsilon, \mu}(x),
$$

where, for any $x \in \Omega \backslash \varepsilon \omega$,

$$
\begin{cases}0 \leq R_{\varepsilon, \mu}(x) \leq c\left(\frac{\varepsilon^{\frac{3 N-2}{4}}}{|x|^{N-2}}+\varepsilon^{\frac{N+2}{4}}\right) & \text { if } N \geq 4 \\ 0 \leq R_{\varepsilon, \mu}(x) \leq c\left(\frac{\varepsilon^{\frac{5}{4}}}{|x|}+\varepsilon^{\frac{3}{4}}\right) & \text { if } N=3\end{cases}
$$

for some positive and fixed constant $c$.

In particular, for any $x \in \Omega \backslash \varepsilon \omega$,

$$
0 \leq U_{\mu, \xi}(x)-P_{\varepsilon} U_{\mu, \xi}(x) \leq c\left(\mu^{\frac{N-2}{2}}+\frac{\varepsilon^{N-2} \mu^{-\left(\frac{N-2}{2}\right)}}{|x|^{N-2}}\right),
$$

for some positive and fixed constant $c$.

Proof. The function $R_{\varepsilon, \mu}$ solves

$$
\begin{cases}-\Delta R_{\varepsilon, \mu}=0 & x \in \Omega_{\varepsilon}, \\ R_{\varepsilon, \mu}(x)=\alpha_{N}\left[-\frac{\mu^{\frac{N-2}{2}}}{\left(\mu^{2}+|x-\xi|^{2}\right)^{\frac{N-2}{2}}}+\frac{\mu^{\frac{N-2}{2}}}{|x-\xi|^{N-2}}+\left(\frac{1}{\mu}\right)^{\frac{N-2}{2}} \varphi_{\omega}\left(\frac{x}{\varepsilon}\right)\right] & x \in \partial \Omega, \\ R_{\varepsilon, \mu}(x)=\alpha_{N}\left[-\frac{\mu^{\frac{N-2}{2}}}{\left(\mu^{2}+|x-\xi|^{2}\right)^{\frac{N-2}{2}}}+\mu^{\frac{N-2}{2}} H(x, \xi)+\left(\frac{1}{\mu}\right)^{\frac{N-2}{2}}\right] & x \in \partial \varepsilon \omega .\end{cases}
$$


Let $\hat{R}_{\varepsilon, \mu}(y):=\mu^{-\frac{N-2}{2}} R_{\varepsilon, \mu}(\varepsilon y), y \in(\Omega / \varepsilon \backslash \omega)$. Then $\hat{R}_{\varepsilon, \mu}$ solves

$$
\begin{cases}-\Delta \hat{R}_{\varepsilon, \mu}=0 & y \in(\Omega / \varepsilon \backslash \omega), \\ \hat{R}_{\varepsilon, \mu}(y)=\alpha_{N}\left[-\frac{1}{\left(\mu^{2}+|\varepsilon y-\xi|^{2}\right)^{\frac{N-2}{2}}}+\frac{1}{|\varepsilon y-\xi|^{N-2}}+\frac{1}{\mu^{N-2}} \varphi_{\omega}(y)\right] & y \in \partial(\Omega / \varepsilon), \\ \hat{R}_{\varepsilon, \mu}(y)=\alpha_{N}\left[-\frac{1}{\left(\mu^{2}+|\varepsilon y-\xi|^{2}\right)^{\frac{N-2}{2}}}+H(\varepsilon y, \xi)+\frac{1}{\mu^{N-2}}\right] & y \in \partial \omega .\end{cases}
$$

In particular, there exists a positive constant $c$ such that

$$
0 \leq \hat{R}_{\varepsilon, \mu}(y) \leq c\left(\mu^{2}+\frac{\varepsilon^{N-2}}{\mu^{N-2}}\right) \leq c\left(\varepsilon+\varepsilon^{\frac{N-2}{2}}\right), y \in \partial(\Omega / \varepsilon) .
$$

On the other hand, using the assumptions that $\mu^{2}=O(\varepsilon)$ and that $\xi=\mu \varepsilon^{\sigma} \tau$ as in (2.1),

$$
0 \leq \hat{R}_{\varepsilon, \mu}(y) \leq c \frac{\varepsilon^{2}}{\mu^{N}} \leq c \frac{1}{\varepsilon^{\frac{N-4}{2}}}, \quad \forall y \in \partial \omega, \text { if } N \geq 4
$$

and

$$
0 \leq \hat{R}_{\varepsilon, \mu}(y) \leq c, \quad \forall y \in \partial \omega, \text { if } N=3 .
$$

Let $R>0$ and $d:=\operatorname{diam} \Omega$. Denote by $\Psi$ the solution to

$$
\begin{cases}-\Delta \Psi=0 & y \in B_{d / \varepsilon} \backslash B_{R} \\ \Psi(y)=\alpha & y \in \partial B_{d / \varepsilon} \\ \Psi(y)=\beta & y \in \partial B_{R}\end{cases}
$$

for some arbitrary $\alpha$ and $\beta$. An easy computation gives that

$$
\Psi(y)=\frac{(\beta-\alpha)(d R)^{N-2}}{d^{N-2}-(\varepsilon R)^{N-2}}\left(\frac{1}{R^{N-2}}-\frac{1}{|y|^{N-2}}\right)+\alpha .
$$

Moreover, for any $y \in B_{d / \varepsilon} \backslash B_{R}$, it holds

$$
0 \leq \Psi(y) \leq \frac{(d R)^{N-2}}{d^{N-2}-(\varepsilon R)^{N-2}}\left(\alpha+\frac{\beta}{|y|^{N-2}}\right) .
$$

Since there are positive numbers $R_{1}$ and $R_{2}$ so that $B\left(0, R_{1}\right) \subset \omega \subset B\left(0, R_{2}\right)$ and since $\Omega / \varepsilon \subset B_{d / \varepsilon}$, by (2.7), (2.8)-(2.13), using maximum principle, we deduce that for any $y \in \Omega / \varepsilon \backslash \omega$,

$$
0 \leq \hat{R}_{\varepsilon, \mu}(y) \leq c\left(\frac{1}{\varepsilon^{\frac{N-4}{2}}|y|^{N-2}}+\varepsilon\right) \quad \text { if } N \geq 4
$$

and

$$
0 \leq \hat{R}_{\varepsilon, \mu}(y) \leq c\left(\frac{1}{|y|}+\sqrt{\varepsilon}\right) \quad \text { if } N=3 .
$$

Therefore, the claim follows. 
The next two lemmas give the size, in terms of $\varepsilon$ and $\mu$, of some integral quantities involving $P_{\varepsilon} U_{\mu, \xi}-U_{\mu, \xi}$ and $R_{\varepsilon, \mu}$. These estimates will be crucial to prove the expansion of the energy functional in Section 5.

Lemma 2.3. Let $\delta>0$ be fixed and assume (2.1). Then

$$
\int_{\Omega \backslash \varepsilon \omega} U_{\mu, \xi}^{p}\left|P_{\varepsilon} U_{\mu, \xi}-U_{\mu, \xi}\right|=O\left(\mu^{N-2}+(\varepsilon / \mu)^{N-2}\right)
$$

and

$$
\int_{\Omega \backslash \varepsilon \omega} U_{\mu, \xi}^{p} R_{\varepsilon, \mu} d x= \begin{cases}O\left(\varepsilon^{N / 2}\right) & \text { if } N \geq 4 \\ O(\varepsilon) & \text { if } N=3,\end{cases}
$$

where the function $R_{\varepsilon, \mu}$ is the one introduced in Lemma 2.2.

Proof. To get the first estimate, in virtue of (2.6), we have to evaluate

$$
\int_{\Omega \backslash \varepsilon \omega} \frac{\mu^{\frac{N+2}{2}}}{\left(\mu^{2}+|x-\xi|^{2}\right)^{\frac{N+2}{2}}}\left(\mu^{\frac{N-2}{2}}+\frac{\varepsilon^{N-2} \mu^{-\left(\frac{N-2}{2}\right)}}{|x|^{N-2}}\right) d x .
$$

It holds (setting $x-\xi=\mu y$ )

$$
\int_{\Omega \backslash \varepsilon \omega} \frac{\mu^{\frac{N+2}{2}}}{\left(\mu^{2}+|x-\xi|^{2}\right)^{\frac{N+2}{2}}} d x=O\left(\mu^{\frac{N-2}{2}} \int_{\mathbb{R}^{N}} \frac{1}{\left(1+|y|^{2}\right)^{\frac{N+2}{2}}} d y\right)
$$

and

$$
\int_{\Omega \backslash \varepsilon \omega} \frac{\mu^{\frac{N+2}{2}}}{\left(\mu^{2}+|x-\xi|^{2}\right)^{\frac{N+2}{2}}} \frac{1}{|x|^{N-2}} d x=O\left(\mu^{-\frac{N-2}{2}} \int_{\mathbb{R}^{N}} \frac{1}{\left(1+|y|^{2}\right)^{\frac{N+2}{2}}} \frac{1}{|y|^{N-2}} d y\right) .
$$

Therefore the first estimate follows. The second one follows by previous estimates and (2.5).

Lemma 2.4. Let $\delta>0$ be fixed and assume (2.1). Then

$$
\int_{\Omega \backslash \varepsilon \omega} U_{\mu, \xi}^{p-1}\left(P_{\varepsilon} U_{\mu, \xi}-U_{\mu, \xi}\right)^{2}= \begin{cases}O\left(\mu^{N}+(\varepsilon / \mu)^{N}\right) & \text { if } N \geq 5, \\ O\left(\mu^{4}|\log \mu|+(\varepsilon / \mu)^{4}|\log (\varepsilon / \mu)|\right) & \text { if } N=4, \\ O\left(\mu^{2}+(\varepsilon / \mu)^{2}\right) & \text { if } N=3 .\end{cases}
$$

Proof. By (2.6), we have to estimate

$$
\int_{\Omega \backslash \varepsilon \omega} \frac{\mu^{2}}{\left(\mu^{2}+|x-\xi|^{2}\right)^{2}}\left(\mu^{N-2}+\frac{\varepsilon^{2(N-2)} \mu^{-(N-2)}}{|x|^{2(N-2)}}\right) d x .
$$


Now, we have if $N \geq 5$

$$
\int_{\Omega \backslash \varepsilon \omega} \frac{\mu^{2}}{\left(\mu^{2}+|x-\xi|^{2}\right)^{2}} d x=0\left(\mu^{2} \int_{\Omega} \frac{1}{|x|^{4}} d x\right)
$$

and if $N=3$ (setting $x-\xi=\mu y$ )

$$
\int_{\Omega \backslash \varepsilon \omega} \frac{\mu^{2}}{\left(\mu^{2}+|x-\xi|^{2}\right)^{2}} d x=0\left(\mu \int_{\mathbb{R}^{N}} \frac{1}{\left(1+|y|^{2}\right)^{2}} d y\right) .
$$

Moreover, we have if $N \geq 5$ (setting $x=\varepsilon y$ )

$$
\int_{\Omega \backslash \varepsilon \omega} \frac{\mu^{2}}{\left(\mu^{2}+|x-\xi|^{2}\right)^{2}} \frac{1}{|x|^{2(N-2)}}=0\left(\varepsilon^{-(N-4)} \mu^{-2} \int_{\{|y| \geq 1\}} \frac{1}{|y|^{2(N-2)}} d y\right)
$$

and if $N=3$ (setting $x-\xi=\mu y$ )

$$
\int_{\Omega \backslash \varepsilon \omega} \frac{\mu^{2}}{\left(\mu^{2}+|x-\xi|^{2}\right)^{2}} \frac{1}{|x|^{2}}=0\left(\mu^{-1} \int_{\mathbb{R}^{N}} \frac{1}{\left(1+|y|^{2}\right)^{2}} \frac{1}{|y|^{2}} d y\right) .
$$

The case $N=4$ can be treated in a similar way.

Collecting all the previous estimates, the claim follows.

\section{Scheme of the proof}

It is useful to perform the following change of variables. Let $\Omega_{\varepsilon}:=\frac{\Omega \backslash \varepsilon \omega}{\sqrt{\varepsilon}}$ and $y=\frac{x}{\sqrt{\varepsilon}} \in \Omega_{\varepsilon}$. Then $u$ is solution to (1.2) if and only if the function

$$
v(y)=\varepsilon^{\frac{1}{p-1}} u(\sqrt{\varepsilon} y)
$$

solves problem

$$
\begin{cases}\Delta v+\varepsilon^{-\frac{\lambda}{p-1}} v^{p+\lambda}=0 & \text { in } \Omega_{\varepsilon}, \\ v>0 & \text { in } \Omega_{\varepsilon}, \\ v=0 & \text { on } \partial \Omega_{\varepsilon} .\end{cases}
$$

In expanded variable, the solution we are looking for looks like $v(y)=V(y)+$ $\phi(y)$, with $V(y)=\varepsilon^{\frac{1}{p-1}} P_{\varepsilon} U_{\mu, \xi}(\sqrt{\varepsilon} y)$ and $\phi(y)=\varepsilon^{\frac{1}{p-1}} \psi(\sqrt{\varepsilon} y)$. Clearly $V$ and $\phi$ depend on $\varepsilon, \mu$ and $\xi$, but for simplicity we drop this dependence in the notation. Beside observe that the function $V$ is nothing but the projection onto $H_{0}^{1}\left(\Omega_{\varepsilon}\right)$ of the function $\varepsilon^{\frac{1}{p-1}} U_{\mu, \xi}(\sqrt{\varepsilon} y)=U_{\frac{\mu}{\sqrt{\varepsilon}}, \xi^{\prime}}(y)$, where we denote by $\xi^{\prime}$ the point $\frac{\xi}{\sqrt{\varepsilon}}$. 
In terms of $\phi$, problem (3.2) becomes

$$
\begin{cases}L(\phi)=-N(\phi)-E & \text { in } \Omega_{\varepsilon}, \\ \phi=0 & \text { on } \partial \Omega_{\varepsilon},\end{cases}
$$

where

$$
\begin{aligned}
& L(\phi):=\Delta \phi+\varepsilon^{-\frac{\lambda}{p-1}}(p+\lambda) V^{p+\lambda-1} \phi, \\
& N(\phi):=\varepsilon^{-\frac{\lambda}{p-1}}\left[(V+\phi)^{p+\lambda}-V^{p+\lambda}-(p+\lambda) V^{p+\lambda-1} \phi\right] \\
& E:=\varepsilon^{-\frac{\lambda}{p-1}} V^{p+\lambda}-U_{d, \xi^{\prime}}^{p} .
\end{aligned}
$$

To prove our result we follow the following strategy. First we solve the problem: given a parameter $\mu>0$ and a point $\xi \in \Omega$ as in (2.1), or equivalently $d>0$ and $\tau \in \mathbb{R}^{N}$, find a function $\phi$, depending on $\tau$ and $d$, such that for certain constants $c_{i}$, depending on $\tau$ and $d, i=0,1, \ldots, N$

$$
\begin{cases}L(\phi)=N(\phi)+E+\sum_{i=0}^{N} c_{i} V^{p-1} Z_{\varepsilon i} & \text { in } \Omega_{\varepsilon} \\ \phi=0 & \text { on } \partial \Omega_{\varepsilon}, \\ \int_{\Omega_{\varepsilon}} V^{p-1} Z_{\varepsilon i} \phi d x=0 & \text { if } i=0,1, \ldots, N .\end{cases}
$$

In order to define the functions $Z_{\varepsilon i}$, we need to recall [6] that the kernel of the operator $-\Delta-p U_{\mu, \xi}^{p-1}$ on $L^{2}\left(\mathbb{R}^{N}\right)$ has dimension $N+1$ and is spanned by the functions

$$
Z_{0}(x):=\frac{\partial U_{\mu, \xi}}{\partial \mu}(x)=\alpha_{N} \frac{N-2}{2} \mu^{(N-4) / 2} \frac{|x-\xi|^{2}-\mu^{2}}{\left(\mu^{2}+|x-\xi|^{2}\right)^{N / 2}}, \quad x \in \mathbb{R}^{N},
$$

and, for $i=1, \ldots, N$,

$$
Z_{i}(x):=\frac{\partial U_{\mu, \xi}}{\partial \xi_{i}}(x)=-\alpha_{N}(N-2) \mu^{(N-2) / 2} \frac{x_{i}-\xi_{i}}{\left(\mu^{2}+|x-\xi|^{2}\right)^{N / 2}}, \quad x \in \mathbb{R}^{N} .
$$

We denote by $P_{\varepsilon} Z_{i}$ the projection onto $\mathrm{H}_{0}^{1}(\Omega \backslash \varepsilon \omega)$ of the function $Z_{i}$, i.e. $\Delta P_{\varepsilon} Z_{i}=$ $\Delta Z_{i}$ in $\Omega \backslash \varepsilon \omega, P_{\varepsilon} Z_{i}=0$ on $\partial(\Omega \backslash \varepsilon \omega)$. We define $Z_{\varepsilon i}(y)=\varepsilon^{\frac{1}{p-1}} P_{\varepsilon} Z_{i}(\sqrt{\varepsilon} y)$ for $y \in \Omega_{\varepsilon}$.

In order to solve problem (3.7), it is necessary to understand first its linear part. Fix $\xi$ and $\mu$ as in (2.1). Given a function $h$, we consider the problem of finding $\phi$ such that for certain real numbers $c_{i}$ the following is satisfied

$$
\begin{cases}L(\phi)=h+\sum_{i=0}^{N} c_{i} V^{p-1} Z_{\varepsilon i} & \text { in } \Omega_{\varepsilon} \\ \phi=0 & \text { on } \partial \Omega_{\varepsilon} \\ \int_{\Omega_{\varepsilon}} V^{p-1} Z_{\varepsilon i} \phi d x=0 & \text { if } i=0,1, \ldots, N\end{cases}
$$


In order to perform an invertibility theory for $L$ subject to the above orthogonality conditions, we introduce $L_{*}^{\infty}\left(\Omega_{\varepsilon}\right)$ and $L_{* *}^{\infty}\left(\Omega_{\varepsilon}\right)$ to be respectively the spaces of functions defined on $\Omega_{\varepsilon}$ with finite $\|\cdot\|_{*}$-norm (respectively $\|\cdot\|_{* *}$-norm), where

$$
\|\psi\|_{*}=\sup _{x \in \Omega_{\varepsilon}}\left[\left|w^{-\beta}(x) \psi(x)\right|+\left|w^{-\left(\beta+\frac{1}{N-2}\right)}(x) D \psi(x)\right|\right],
$$

with

$$
w(x)=\left(1+\left|x-\xi^{\prime}\right|^{2}\right)^{-\frac{N-2}{2}}, \quad \xi^{\prime}=\frac{\xi}{\sqrt{\varepsilon}},
$$

$\beta=1$ if $N=3$ and $\beta=\frac{2}{N-2}$ if $N \geq 4$. Similarly we define, for any dimension $N \geq 3$,

$$
\|\psi\|_{* *}=\sup _{x \in \Omega_{\varepsilon}}\left|w^{-\frac{4}{N-2}}(x) \psi(x)\right| .
$$

Indeed the operator $L$ is uniformly invertible with respect to the above weighted $L^{\infty}$-norm, for all $\varepsilon$ small enough. This fact is established in next Proposition.

Proposition 3.1. Let $\delta>0$ be fixed. There are numbers $\varepsilon_{0}>0, C>0$, such that, for points $\xi$ and parameters $\mu$ satisfying (2.1) problem (3.8) admits a unique solution $\phi \equiv T(h)$ for all $0<\varepsilon<\varepsilon_{0}$ and all $h \in C^{\alpha}\left(\bar{\Omega}_{\varepsilon}\right)$. Besides,

$$
\|T(h)\|_{*} \leq C\|h\|_{* *}, \quad\left\|\partial_{\xi^{\prime}, d} T(h)\right\|_{*} \leq C\|h\|_{* *}
$$

and

$$
\left|c_{i}\right| \leq C\|h\|_{* *} .
$$

Next step is the finite dimensional reduction: we consider the nonlinear problem of finding a function $\phi$ such that for some constants $c_{i}$ the following equation holds

$$
\begin{cases}\Delta(V+\phi)+e^{-\frac{\lambda}{p-1}}(V+\phi)_{+}^{p+\lambda}=\sum_{i} c_{i} V^{p-1} Z_{\varepsilon i} & \text { in } \Omega_{\varepsilon}, \\ \phi=0 & \text { on } \partial \Omega_{\varepsilon}, \\ \int_{\Omega_{\varepsilon}} \phi V^{p-1} Z_{\varepsilon i}=0 & \text { for all } i .\end{cases}
$$

The solvability of problem (3.11) is established in next Proposition, whose proof is postponed to Section 6.

Proposition 3.2. Assume the conditions of Proposition 3.1 are satisfied. Then there is a $C>0$, such that for all small $\varepsilon$ there exists a unique solution $\phi=\phi\left(\xi^{\prime}, d\right)$ to problem (3.11), such that the map $\left(\xi^{\prime}, d\right) \rightarrow \phi\left(\xi^{\prime}, d\right)$ is of class $C^{1}$ for $\|\cdot\|_{*}$-norm and

$$
\begin{array}{r}
\|\phi\|_{*} \leq C\left(|\lambda \log \varepsilon|+\varepsilon^{\frac{N-2}{2}}\right), \\
\left\|\nabla_{\left(\xi^{\prime}, d\right)} \phi\right\|_{*} \leq C\left(|\lambda \log \varepsilon|+\varepsilon^{\frac{N-2}{2}}\right) .
\end{array}
$$


Now, we can reduce the problem to a finite dimensional one.

Let us consider now the functional $J_{\varepsilon}: \mathbb{R}^{N} \times(0,+\infty) \rightarrow+\infty$ defined by

$$
J_{\varepsilon}(\tau, d)=I_{\varepsilon}(V+\phi)
$$

where $\phi$ is the function given by Proposition 3.2 and $I_{\varepsilon}$ is given by

$$
I_{\varepsilon}(v)=\frac{1}{2} \int_{\Omega_{\varepsilon}}|D v|^{2}-\frac{\varepsilon^{-\frac{\lambda}{p-1}}}{p+\lambda+1} \int_{\Omega_{\varepsilon}} v^{p+\lambda+1} .
$$

Lemma 3.3. $v=V+\phi$ is a solution of problem (3.2), namely $c_{i}=0$ in (3.11) for all $i$, if and only if $(\tau, d)$ is a critical point of $J_{\varepsilon}$.

Proof. The definition of $\phi$ yields that $I_{\varepsilon}^{\prime}(V+\phi)[\eta]=0$ for all $\eta$ which vanishes on $\partial \Omega_{\varepsilon}$ and such that $\int_{\Omega_{\varepsilon}} \eta V^{p-1} Z_{\varepsilon i}=0$ for all $i$. It is easy to check that $\frac{\partial V}{\partial \xi_{j}^{\prime}}=$ $Z_{\varepsilon j}+o(1), \quad \frac{\partial V}{\partial d}=Z_{\varepsilon 0}+o(1)$, with $o(1)$ small as $\varepsilon \rightarrow 0$. This fact, together with the last part of Proposition 3.2, proves our claim (see [12] for more details).

It is crucial to compute the expansion of $J_{\varepsilon}$ with respect to $\varepsilon$.

Proposition 3.4. Let $\delta>0$ be fixed and assume (2.1) and (2.2) hold true. Then we have the following expansion

$$
J_{\varepsilon}(\tau, d)=a_{1}+\Psi(\tau, d) \varepsilon^{\frac{N-2}{2}}+\frac{1}{2} b_{2} \Lambda \varepsilon^{\frac{N-2}{2}} \log \varepsilon+o\left(\varepsilon^{\frac{N-2}{2}}\right),
$$

where

$$
\Psi(\tau, d):=a_{2}|\tau|^{2}-a_{3} H(0,0) d^{N-2}-a_{4} \frac{1}{d^{N-2}}+b_{1} \Lambda+b_{2} \Lambda \log d,
$$

$a_{1}, \ldots, a_{4}$ and $b_{1}, b_{2}$ are positive constans and, as $\varepsilon$ goes to zero, the term o $\left(\varepsilon^{\frac{N-2}{2}}\right)$ is $C^{1}$ uniform over all d's and $\tau$ 's satisfying (2.1).

Proof. Firstly, we observe that, under the change of variables (3.1), we have that

$$
I_{\varepsilon}(v)=J_{\varepsilon, \lambda}(u), \quad J_{\varepsilon, \lambda}(u)=\frac{1}{2} \int_{\Omega \backslash \varepsilon \omega}|D v|^{2}-\frac{\varepsilon^{-\frac{\lambda}{p-1}}}{p+\lambda+1} \int_{\Omega \backslash \varepsilon \omega} v^{p+\lambda+1} .
$$

Hence the function $P_{\varepsilon} U_{\mu, \xi}+\psi$, with $\psi(x)=\varepsilon^{-\frac{1}{p-1}} \phi\left(\frac{x}{\sqrt{\varepsilon}}\right)$, is a solution to (1.2) if and only if $(d, \tau)$ is a critical point of the function

$$
J_{\varepsilon}(\tau, d)=J_{\varepsilon, \lambda}\left(P_{\varepsilon} U_{\mu, \xi}+\phi\right) .
$$

Therefore, the claim follows by Lemma 4.3, Lemma 2.2 and Lemma 4.2, taking in account (2.1), (2.2) and the fact

$$
\begin{aligned}
& J_{\varepsilon, \lambda}\left(P_{\varepsilon} U_{\mu, \xi}\right)=J_{\varepsilon, 0}\left(P_{\varepsilon} U_{\mu, \xi}\right) \\
& -\left(\frac{1}{p+1+\lambda} \int_{\Omega \backslash \varepsilon \omega}\left(P_{\varepsilon} U_{\mu, \xi}\right)^{p+1+\lambda}-\frac{1}{p+1} \int_{\Omega \backslash \varepsilon \omega}\left(P_{\varepsilon} U_{\mu, \xi}\right)^{p+1}\right) .
\end{aligned}
$$


Proof of Theorem 1.1. In virtue of Lemma 3.3 and Lemma 3.4, we need to find a critical point of the function $\Psi$, defined in (3.17), which is stable under $C^{1}$-perturbation. On the other hand, it is easy to check that, if $\Lambda$ is any real number, possibly depending on $\varepsilon$ and bounded from above and from below, the function $\Psi$ has a non degenerate critical point of "saddle" type, which is stable with respect to $C^{1}$-perturbation. Therefore, the claim is proved.

\section{The expansion of the energy}

Lemma 4.1. Let $\delta>0$ be fixed and assume (2.1). Then

$$
J_{\varepsilon, 0}\left(P_{\varepsilon} U_{\mu, \xi}\right)=a_{1}+a_{2}|\tau|^{2} \varepsilon^{2 \sigma}-a_{3} H(0,0) \mu^{N-2}(1+o(1))-a_{4}\left(\frac{\varepsilon}{\mu}\right)^{N-2}(1+o(1)),
$$

uniformly in $C^{1}$ sense for $\tau$ and $d$ satisfying (2.1). The constants that appear in (4.1) are given by

$$
\begin{aligned}
& a_{1}:=\frac{1}{N} \alpha_{N}^{p+1} \int_{\mathbb{R}^{N}} \frac{1}{\left(1+|y|^{2}\right)^{N}} d y, \\
& a_{2}:=\alpha_{N}^{p+1} \int_{\mathbb{R}^{N}}\left[2 N(N+1) \frac{y_{1}^{2}}{\left(1+|y|^{2}\right)^{N+2}}-\frac{1}{\left(1+|y|^{2}\right)^{N+1}}\right] d y, \\
& a_{3}:=\frac{1}{2} \alpha_{N}^{p+1} \int_{\mathbb{R}^{N}} \frac{1}{\left(1+|y|^{2}\right)^{\frac{N+2}{2}}} d y, \\
& a_{4}:=\frac{1}{2} \alpha_{N}^{p+1} c_{\omega} \int_{\mathbb{R}^{N}} \frac{1}{|y|^{N-2}} \frac{1}{\left(1+|y|^{2}\right)^{\frac{N+2}{2}}} d y, .
\end{aligned}
$$

Proof. Via a Taylor expansion, we have (for some $t \in(0,1)$ )

$$
\begin{aligned}
J_{\varepsilon, 0}\left(P_{\varepsilon} U_{\mu, \xi}\right)= & \frac{1}{2} \int_{\Omega \backslash \varepsilon \omega}\left|\nabla P_{\varepsilon} U_{\mu, \xi}\right|^{2}-\frac{1}{p+1} \int_{\Omega \backslash \varepsilon \omega}\left(P_{\varepsilon} U_{\mu, \xi}\right)^{p+1} \\
= & \frac{1}{2} \int_{\Omega \backslash \varepsilon \omega} P_{\varepsilon} U_{\mu, \xi} U_{\mu, \xi}^{p}-\frac{1}{p+1} \int_{\Omega \backslash \varepsilon \omega}\left(P_{\varepsilon} U_{\mu, \xi}\right)^{p+1} \\
= & \frac{1}{N} \int_{\Omega \backslash \varepsilon \omega} U_{\mu, \xi}^{p+1}-\frac{1}{2} \int_{\Omega \backslash \varepsilon \omega}\left(P_{\varepsilon} U_{\mu, \xi}-U_{\mu, \xi}\right) U_{\mu, \xi}^{p} \\
& -\frac{p}{2} \int_{\Omega \backslash \varepsilon \omega}\left(t P_{\varepsilon} U_{\mu, \xi}+(1-t) U_{\mu, \xi}\right)^{p-1}\left(P_{\varepsilon} U_{\mu, \xi}-U_{\mu, \xi}\right)^{2}
\end{aligned}
$$


Now, it holds (setting $x=\mu y$ )

$$
\begin{aligned}
\int_{\Omega \backslash \varepsilon \omega} U_{\mu, \xi}^{p+1} & =\alpha_{N}^{p+1} \int_{\Omega \backslash \varepsilon \omega} \frac{\mu^{N}}{\left(\mu^{2}+|x-\xi|^{2}\right)^{N}} d x \\
& =\alpha_{N}^{p+1} \int_{\frac{\Omega \backslash \varepsilon \omega}{\mu}} \frac{1}{\left(1+\left|y-\varepsilon^{\sigma} \tau\right|^{2}\right)^{N}} d y \\
& =\alpha_{N}^{p+1} \int_{\mathbb{R}^{N}} \frac{1}{\left(1+\left|y-\varepsilon^{\sigma} \tau\right|^{2}\right)^{N}} d y+O\left(\left(\frac{\varepsilon}{\mu}\right)^{N}+\mu^{N}\right) .
\end{aligned}
$$

Moreover, via a Taylor expansion we get

$$
\begin{aligned}
\frac{1}{\left(1+\left|y-\varepsilon^{\sigma} \tau\right|^{2}\right)^{N}}= & \frac{1}{\left(1+|y|^{2}\right)^{N}}+2 N \frac{(y, \tau)}{\left(1+|y|^{2}\right)^{N+1}} \varepsilon^{\sigma} \\
& +\left[2 N(N+1) \frac{(y, \tau)^{2}}{\left(1+|y|^{2}\right)^{N+2}}-N \frac{|\tau|^{2}}{\left(1+|y|^{2}\right)^{N+1}}\right] \varepsilon^{2 \sigma} \\
& +R(\varepsilon, y) \varepsilon^{3 \sigma}
\end{aligned}
$$

where

$$
|R(\varepsilon, y)| \leq c \frac{|y|}{\left(1+|y|^{2}\right)^{N+2}}, y \in \mathbb{R}^{N} .
$$

By (4.8) and (4.9), we deduce that

$$
\begin{aligned}
& \int_{\mathbb{R}^{N}} \frac{1}{\left(1+\left|y-\varepsilon^{\sigma} \tau\right|^{2}\right)^{N}} d y=\int_{\mathbb{R}^{N}} \frac{1}{\left(1+|y|^{2}\right)^{N}} d y \\
& +2 N \varepsilon^{\sigma} \int_{\mathbb{R}^{N}} \frac{(y, \tau)}{\left(1+|y|^{2}\right)^{N+1}} d y \\
& +\varepsilon^{2 \sigma} \int_{\mathbb{R}^{N}}\left[2 N(N+1) \frac{(y, \tau)^{2}}{\left(1+|y|^{2}\right)^{N+2}}-N \frac{|\tau|^{2}}{\left(1+|y|^{2}\right)^{N+1}}\right] d y \\
& +O\left(\varepsilon^{3 \sigma}\right) \\
& =\int_{\mathbb{R}^{N}} \frac{1}{\left(1+|y|^{2}\right)^{N}} d y \\
& +\varepsilon^{2 \sigma}|\tau|^{2} \int_{\mathbb{R}^{N}}\left[2 N^{2}(N+1) \frac{y_{1}^{2}}{\left(1+|y|^{2}\right)^{N+2}}-N \frac{1}{\left(1+|y|^{2}\right)^{N+1}}\right] d y \\
& +O\left(\varepsilon^{3 \sigma}\right) \text {. }
\end{aligned}
$$


Thus we obtain the first two terms in (4.1) with constants $a_{1}$ and $a_{2}$ given respectively by (4.2) and (4.3). An easy computation shows that $a_{2}>0$.

We have, by Lemma 2.2

$$
\begin{aligned}
& \int_{\Omega \backslash \varepsilon \omega}\left(P_{\varepsilon} U_{\mu, \xi}-U_{\mu, \xi}\right) U_{\mu, \xi}^{p} d x \\
& =-\alpha_{N}^{p+1} \int_{\Omega \backslash \varepsilon \omega}\left(\mu^{\frac{N-2}{2}} H(x, \xi)+\frac{1}{\mu^{\frac{N-2}{2}}} \varphi_{\omega}\left(\frac{x}{\varepsilon}\right)\right) \frac{\mu^{\frac{N+2}{2}}}{\left(\mu^{2}+|x-\xi|^{2}\right)^{\frac{N+2}{2}}} d x \\
& \quad+\int_{\Omega \backslash \varepsilon \omega} R_{\varepsilon, \mu} U_{\mu, \xi}^{p} d x .
\end{aligned}
$$

Now, (setting $x-\xi=\mu y$ ) we have

$$
\begin{aligned}
\int_{\Omega \backslash \varepsilon \omega} & \mu^{\frac{N-2}{2}} H(x, \xi) \frac{\mu^{\frac{N+2}{2}}}{\left(\mu^{2}+|x-\xi|^{2}\right)^{\frac{N+2}{2}}} d x \\
= & \int_{\frac{\Omega \backslash \varepsilon \omega-\xi}{\mu}} \mu^{N-2} H(\mu y+\xi, \xi) \frac{1}{\left(1+|y|^{2}\right)^{\frac{N+2}{2}}} d y \\
= & \mu^{N-2} H(0,0)\left(\int_{\mathbb{R}^{N}} \frac{1}{\left(1+|y|^{2}\right)^{\frac{N+2}{2}}} d y+o(1)\right) .
\end{aligned}
$$

The previous formula computes the third term in the expansion given by (4.1). Moreover, we get

$$
\begin{aligned}
\frac{1}{\mu^{\frac{N-2}{2}}} & \int_{\Omega \backslash \varepsilon \omega} \varphi_{\omega}\left(\frac{x}{\varepsilon}\right) \frac{\mu^{\frac{N+2}{2}}}{\left(\mu^{2}+|x-\xi|^{2}\right)^{\frac{N+2}{2}}} d x \\
& =\int_{\frac{\Omega \backslash \varepsilon \omega-\xi}{\mu}} \varphi_{\omega}\left(\frac{\mu}{\varepsilon}\left(y+\varepsilon^{\sigma} \tau\right)\right) \frac{1}{\left(1+|y|^{2}\right)^{\frac{N+2}{2}}} d y \\
& =\left(\frac{\varepsilon}{\mu}\right)^{N-2} \int_{\frac{\Omega \backslash \varepsilon \omega-\xi}{\mu}} f_{\varepsilon}(y) \frac{1}{\left|y+\varepsilon^{\sigma} \tau\right|^{N-2}} \frac{1}{\left(1+|y|^{2}\right)^{\frac{N+2}{2}}} d y \\
& =\left(\frac{\varepsilon}{\mu}\right)^{N-2}\left(c_{\omega} \int_{\mathbb{R}^{N}} \frac{1}{|y|^{N-2}} \frac{1}{\left(1+|y|^{2}\right)^{\frac{N+2}{2}}} d y+o(1)\right) .
\end{aligned}
$$


Here, we set $f_{\varepsilon}(y):=\left(\frac{\mu}{\varepsilon}\right)^{N-2}\left|y+\varepsilon^{\sigma} \tau\right|^{N-2} \varphi_{\omega}\left(\frac{\mu}{\varepsilon}\left(y+\varepsilon^{\sigma} \tau\right)\right)$ and we apply Lebesgue's dominated convergence theorem and Lemma 2.1. This fact gives the fourth term in the expansion (4.1).

Collecting all the previous estimates and taking into account Lemma 2.3 and Lemma 2.4, we get the claim.

Lemma 4.2. Let $\delta>0$ be fixed and assume (2.1), (2.2). Then

$$
\begin{gathered}
\frac{1}{p+1+\lambda} \int_{\Omega \backslash \varepsilon \omega}\left(P_{\varepsilon} U_{\mu, \xi}\right)^{p+1+\lambda}-\frac{1}{p+1} \int_{\Omega \backslash \varepsilon \omega}\left(P_{\varepsilon} U_{\mu, \xi}\right)^{p+1} \\
=-\left(b_{1}+b_{2} \log \mu+o(1)\right) \lambda,
\end{gathered}
$$

uniformly in $C^{1}$ sense for $\tau$ and d satisfying (2.1). Here

$$
\begin{aligned}
b_{1}:= & \frac{1}{(p+1) 2} \alpha_{N}^{p+1} \int_{\mathbb{R}^{N}} \frac{1}{\left(1+|y|^{2}\right)^{N}} d y \\
& -\frac{1}{p+1} \alpha_{N}^{p+1} \int_{\mathbb{R}^{N}} \frac{1}{\left(1+|y|^{2}\right)^{N}} \log \frac{1}{\left(1+|y|^{2}\right)^{\frac{N-2}{2}}} d y, \\
b_{2}:= & \frac{N-2}{2(p+1)} \alpha_{N}^{p+1} \int_{\mathbb{R}^{N}} \frac{1}{\left(1+|y|^{2}\right)^{N}} d y .
\end{aligned}
$$

Proof. Via a Taylor expansion, we have (for some $t \in(0,1)$ )

$$
\begin{aligned}
& \frac{1}{p+1+\lambda} \int_{\Omega \backslash \varepsilon \omega}\left(P_{\varepsilon} U_{\mu, \xi}\right)^{p+1+\lambda}-\frac{1}{p+1} \int_{\Omega \backslash \varepsilon \omega}\left(P_{\varepsilon} U_{\mu, \xi}\right)^{p+1} \\
= & \lambda\left[-\frac{1}{(p+1)^{2}} \int_{\Omega \backslash \varepsilon \omega}\left(P_{\varepsilon} U_{\mu, \xi}\right)^{p+1}+\frac{1}{p+1} \int_{\Omega \backslash \varepsilon \omega}\left(P_{\varepsilon} U_{\mu, \xi}\right)^{p+1} \log P_{\varepsilon} U_{\mu, \xi}\right] \\
+ & \frac{1}{2} \lambda^{2} \int_{\Omega \backslash \varepsilon \omega}\left(\frac{2}{(p+1+t \lambda)^{3}}-\frac{2 \log P_{\varepsilon} U_{\mu, \xi}}{(p+1+t \lambda)^{2}}+\frac{\log ^{2} P_{\varepsilon} U_{\mu, \xi}}{p+1+t \lambda}\right)\left(P_{\varepsilon} U_{\mu, \xi}\right)^{p+1+t \lambda} .
\end{aligned}
$$

Let $R(\lambda)$ be the last integral in formula above. Using estimate (2.6), it is not difficult to check that

$$
|R(\lambda)| \leq c|\log \mu|^{2} \text { if } \lambda \leq 0,|R(\lambda)| \leq c \mu^{-\lambda}|\log \mu|^{2} \text { if } \lambda>0 .
$$


Moreover, via a Taylor expansion, we have (for some $t \in(0,1)$ )

$$
\begin{aligned}
& -\frac{1}{(p+1)^{2}} \int_{\Omega \backslash \varepsilon \omega}\left(P_{\varepsilon} U_{\mu, \xi}\right)^{p+1} d x+\frac{1}{p+1} \int_{\Omega \backslash \varepsilon \omega}\left(P_{\varepsilon} U_{\mu, \xi}\right)^{p+1} \log P_{\varepsilon} U_{\mu, \xi} d x \\
= & -\frac{1}{(p+1)^{2}} \int_{\Omega \backslash \varepsilon \omega} U_{\mu, \xi}^{p+1} d x+\frac{1}{p+1} \int_{\Omega \backslash \varepsilon \omega} U_{\mu, \xi}^{p+1} \log U_{\mu, \xi} d x \\
& -\frac{1}{(p+1)} \int_{\Omega \backslash \varepsilon \omega}\left(U_{\mu, \xi}+t P_{\varepsilon} U_{\mu, \xi}\right)^{p}\left(P_{\varepsilon} U_{\mu, \xi}-U_{\mu, \xi}\right) d x \\
& +\int_{\Omega \backslash \varepsilon \omega}\left[\left(U_{\mu, \xi}+t P_{\varepsilon} U_{\mu, \xi}\right)^{p} \log \left(U_{\mu, \xi}+t P_{\varepsilon} U_{\mu, \xi}\right)+\frac{1}{(p+1)}\left(U_{\mu, \xi}+t P_{\varepsilon} U_{\mu, \xi}\right)^{p}\right] \\
= & -\frac{1}{(p+1)^{2}} \int_{\mathbb{R}^{N}} U^{p+1} d y+\frac{1}{p+1} \int_{\mathbb{R}^{N}} U^{p+1}\left(-\log \mu^{\frac{N-2}{2}}+\log U\right) d y+o(1),
\end{aligned}
$$

where $U(y)=\alpha_{N} /\left(1+|y|^{2}\right)^{\frac{N-2}{2}}$. Here, we used Lemma 2.4, Lemma 2.3 and estimate (2.6). Therefore, the claim follows.

The following estimate is crucial to find critical points of $J_{\varepsilon}$.

Lemma 4.3. Let $\delta>0$ be fixed. Assume (2.1) and (2.2) hold true. Then we have

$$
J_{\varepsilon, \lambda}\left(P_{\varepsilon} U_{\mu, \xi}+\phi\right)=J_{\varepsilon, \lambda}\left(P_{\varepsilon} U_{\mu, \xi}\right)+o\left(\varepsilon^{\frac{N-2}{2}}\right)
$$

where as $\varepsilon$ goes to zero the term o $\left(\varepsilon^{\frac{N-2}{2}}\right)$ is $C^{1}$ uniform over all $d$ 's and $\tau$ 's satisfying (2.1).

Proof. We show that (using notation in (3.18) and in (3.18))

$$
J_{\varepsilon}(\tau, d)-I_{\varepsilon}(V)=o\left(\varepsilon^{\frac{N-2}{2}}\right)
$$

and

$$
\nabla_{\tau, d}\left[J_{\varepsilon}(\tau, d)-I_{\varepsilon}(V)\right]=o\left(\varepsilon^{\frac{N-2}{2}}\right)
$$

The conclusion follows from the fact that $I_{\varepsilon}(V)=J_{\varepsilon, \lambda}\left(P_{\varepsilon} U_{\mu, \xi}\right)$. 
Taking into account that $0=D I_{\varepsilon}(V+\tilde{\psi}+\tilde{\phi})[\tilde{\phi}]$, a Taylor expansion gives

$$
\begin{aligned}
& I_{\varepsilon}(V+\tilde{\psi})-J_{\varepsilon}(\tau, d)=\int_{0}^{1} t d t D^{2} I_{\varepsilon}(V+\tilde{\psi}+t \tilde{\phi})[\tilde{\phi}, \tilde{\phi}] \\
& =\int_{0}^{1} t d t\left[\int_{\Omega_{\varepsilon}}|\nabla \tilde{\phi}|^{2}-(p+\lambda)(V+\tilde{\psi}+t \tilde{\phi})^{p+\lambda-1} \tilde{\phi}^{2}\right] \\
& =\int_{0}^{1} t d t\left(\int_{\Omega_{\varepsilon}} N(\tilde{\phi}+\tilde{\psi}) \tilde{\phi}+\int_{\Omega_{\varepsilon}}(p+\lambda)\left[V^{p+\lambda-1}-(V+\tilde{\psi}+t \tilde{\phi})^{p+\lambda-1}\right] \tilde{\phi}^{2}\right) .
\end{aligned}
$$

Since $\|\tilde{\phi}\|_{*}=O\left(|\lambda \log \varepsilon|+\varepsilon^{\frac{N-2}{2}}\right)$, we get

$$
J_{\varepsilon}(\tau, d)-I_{\varepsilon}(V+\tilde{\psi})=O\left(\left(|\lambda \log \varepsilon|+\varepsilon^{\frac{N-2}{2}}\right)^{2}\right)=o\left(\varepsilon^{\frac{N-2}{2}}\right) .
$$

Differentiating with respect to $\tau$ variables we get from (2.1) and form (4.21) that

$$
\begin{aligned}
& D_{\tau}\left[I_{\varepsilon}(V+\tilde{\psi})-J_{\varepsilon}(\tau, d)\right]=\mu \varepsilon^{\sigma} D_{\xi}\left[I_{\varepsilon}(V+\tilde{\psi})-J_{\varepsilon}(\tau, d)\right] \\
& =\mu \varepsilon^{\sigma} \varepsilon^{-\frac{1}{2}} \int_{0}^{1} t d t\left(\int_{\Omega_{\varepsilon}} D_{\xi^{\prime}}[(N(\tilde{\phi}+\tilde{\psi})) \tilde{\phi}]\right. \\
& \left.\quad+(p+\lambda) \int_{\Omega_{\varepsilon}} D_{\xi^{\prime}}\left[\left((V+\tilde{\psi}+t \tilde{\phi})^{p+\lambda-1}-(V+\tilde{\psi})^{p+\lambda-1}\right) \tilde{\phi}^{2}\right]\right) .
\end{aligned}
$$

Using the computations in the proof of Proposition 3.2 we get that the first integral in relation (4.23) can be estimated by $O\left(\left(|\lambda \log \varepsilon|+\varepsilon^{\frac{N-2}{2}}\right)^{2}\right)$, so does the second; hence

$$
D_{\tau}\left[J_{\varepsilon}(\tau, d)-I_{\varepsilon}(V+\tilde{\psi})\right]=o\left(\varepsilon^{\frac{N-2}{2}}\right) .
$$

Now, since $D I_{\varepsilon}(V)[\tilde{\psi}]=\int_{\Omega_{\varepsilon}} R \tilde{\psi}$, we have

$$
\begin{aligned}
& I_{\varepsilon}(V+\tilde{\psi})-I_{\varepsilon}(V) \\
& =\left\{\int_{0}^{1}(1-t) d t\left[(p+\lambda) \int_{\Omega_{\varepsilon}}\left((V+t \tilde{\psi})^{p+\lambda-1}-V^{p+\lambda-1}\right) \tilde{\psi}^{2}\right]-2 \int_{\Omega_{\varepsilon}} R \tilde{\psi}\right\} .
\end{aligned}
$$

Since $\|\tilde{\psi}\|_{*}+\|R\|_{* *}=O\left(|\lambda \log \varepsilon|+\varepsilon^{\frac{N-2}{2}}\right)$, the above term is $O\left(\left(|\lambda \log \varepsilon|+\varepsilon^{\frac{N-2}{2}}\right)^{2}\right)$; then, (4.19) follows from (4.22) and (4.24). Using again (4.24), we see that

$$
\begin{aligned}
& D_{\tau}\left[I_{\varepsilon}(V+\tilde{\psi})-I_{\varepsilon}(V)\right]=\mu \varepsilon^{\sigma} D_{\xi}\left[I_{\varepsilon}(V+\tilde{\psi})-I_{\varepsilon}(V)\right] \\
& =\mu \varepsilon^{\sigma} \varepsilon^{-\frac{1}{2}} D_{\xi^{\prime}}\left\{\int_{0}^{1}(1-t) d t\left[(p+\lambda) \int_{\Omega_{\varepsilon}}\left((V+t \tilde{\psi})^{p+\lambda-1}-V^{p+\lambda-1}\right) \tilde{\psi}^{2}\right]-2 \int_{\Omega_{\varepsilon}} R \tilde{\psi}\right\} .
\end{aligned}
$$


Since from Proposition 3.2 it follows that $\left\|D_{\xi^{\prime}} \tilde{\psi}\right\|_{*}=O\left(\left(|\lambda \log \varepsilon|+\varepsilon^{\frac{N-2}{2}}\right)\right)$, we get

$$
D_{\tau}\left[I_{\varepsilon}(V+\tilde{\psi})-I_{\varepsilon}(V)\right]=O\left(|\lambda|^{2} \mu \varepsilon^{\sigma-\frac{1}{2}}\right)=o\left(\varepsilon^{\frac{N-2}{2}}\right) .
$$

This concludes the proof.

\section{The linear problem: proof of Proposition 3.1}

The proof of this Proposition consists of 2 steps.

Step 1. Assume there exists a sequence $\varepsilon=\varepsilon_{n} \rightarrow 0$ such that there are functions $\phi_{\varepsilon}$ and $h_{\varepsilon}$ with $\left\|h_{\varepsilon}\right\|_{* *}=o(1)$, such that

$$
\begin{cases}L\left(\phi_{\varepsilon}\right)=h_{\varepsilon}+\sum_{i=0}^{N} c_{i} V^{p-1} Z_{\varepsilon i} & \text { in } \Omega_{\varepsilon}, \\ \phi_{\varepsilon}=0 & \text { on } \partial \Omega_{\varepsilon}, \\ \int_{\Omega_{\varepsilon}} V^{p-1} Z_{\varepsilon i} \phi_{\varepsilon} d x=0 & \text { if } i=0,1, \ldots, N,\end{cases}
$$

for certain constants $c_{i}$, depending on $\varepsilon$. Then

$$
\left\|\phi_{\varepsilon}\right\|_{*} \rightarrow 0
$$

We shall establish first the slightly weaker assertion that

$$
\left\|\phi_{\varepsilon}\right\|_{\rho}=\sup _{x \in \Omega_{\varepsilon}}\left[\left|w^{-(\beta-\rho)} \phi_{\varepsilon}(x)\right|+\left|w^{-\left(\beta-\rho+\frac{1}{N-2}\right)} D \phi_{\varepsilon}(x)\right|\right] \rightarrow 0
$$

with $\rho>0$ a small fixed number. To do this, we assume the opposite, so that with no loss of generality we may take $\left\|\phi_{\varepsilon}\right\|_{\rho}=1$. Testing the above equation (5.1) against $Z_{\varepsilon l}$, integrating by parts twice we get that

$$
\sum c_{i} \int_{\Omega_{\varepsilon}} V^{p-1} Z_{\varepsilon i} Z_{\varepsilon l}=\int_{\Omega_{\varepsilon}}\left[\Delta Z_{\varepsilon l}+(p+\lambda) V^{p-1+\lambda} Z_{\varepsilon l}\right] \phi_{\varepsilon}-\int_{\Omega_{\varepsilon}} h_{\varepsilon} Z_{\varepsilon l} .
$$

This defines a linear system in the $c_{i}$ 's which is "almost diagonal" as $\varepsilon$ approaches zero, since we have for $l=1, \ldots, N$

$$
\int_{\Omega_{\varepsilon}} V^{p-1} Z_{\varepsilon i} Z_{\varepsilon l}=\delta_{i, l} \int_{\mathbb{R}^{N}} U_{\Lambda_{i}, 0}^{p-1}\left(\frac{\partial U_{\Lambda_{i}, 0}}{\partial x_{l}}\right)^{2}+o(1)
$$

and for $l=0$

$$
\int_{\Omega_{\varepsilon}} V^{p-1} Z_{\varepsilon i} Z_{\varepsilon 0}=\delta_{i, 0} \int_{\mathbb{R}^{N}} U_{\Lambda_{i}, 0}^{p-1}\left(\frac{\partial U_{\Lambda_{i}, 0}}{\partial \Lambda_{i}}\right)^{2}+o(1)
$$

for suitable $\Lambda_{i}>0$. 
On the other hand, it is easy to see that

$$
\int_{\Omega_{\varepsilon}}\left[\Delta Z_{\varepsilon l}+(p+\lambda) V^{p+\lambda-1} Z_{\varepsilon l}\right] \phi_{\varepsilon}=o(1)\left\|\phi_{\varepsilon}\right\|_{\rho},
$$

and

$$
\left|\int_{\Omega_{\varepsilon}} h_{\varepsilon} Z_{\varepsilon l}\right| \leq C\left\|h_{\varepsilon}\right\|_{* *}
$$

Thus, we conclude that

$$
\left|c_{i}\right| \leq C\left\|h_{\varepsilon}\right\|_{* *}+o(1)\left\|\phi_{\varepsilon}\right\|_{\rho}
$$

so that $c_{i}=o(1)$. Now we can rewrite the equation in the following form

$$
\begin{aligned}
\phi_{\varepsilon}(x) & -(p+\lambda) \int_{\Omega_{\varepsilon}} G_{\varepsilon}(x, y) V^{p+\lambda-1} \phi_{\varepsilon} d y \\
& =-\int_{\Omega_{\varepsilon}} G_{\varepsilon}(x, y) h_{\varepsilon} d y-\sum_{i} c_{i} \int_{\Omega_{\varepsilon}} V^{p-1} Z_{\varepsilon i} G_{\varepsilon}(x, y) d y \quad x \in \Omega_{\varepsilon},
\end{aligned}
$$

where $G_{\varepsilon}$ denotes the Green's function of $\Omega_{\varepsilon}$. Furthermore, the function $\phi_{\varepsilon}$ is of class $C 1$ and

$$
\begin{aligned}
& \partial_{x_{j}} \phi_{\varepsilon}(x)-(p+\lambda) \int_{\Omega_{\varepsilon}} \partial_{x_{j}} G_{\varepsilon}(x, y) V^{p+\lambda-1} \phi_{\varepsilon} d y \\
& =-\int_{\Omega_{\varepsilon}} \partial_{x_{j}} G_{\varepsilon}(x, y) h_{\varepsilon} d y-\sum_{i} c_{i} \int_{\Omega_{\varepsilon}} V^{p-1} Z_{\varepsilon i} \partial_{x_{j}} G_{\varepsilon}(x, y) d y \quad x \in \Omega_{\varepsilon} .
\end{aligned}
$$

We make now the following observation

$$
\begin{aligned}
\int_{\Omega_{\varepsilon}}\left|G_{\varepsilon}(x, y) h_{\varepsilon}\right| d y & \leq\left\|h_{\varepsilon}\right\|_{* *} C \int_{\mathbb{R}^{N}} \Gamma(x-y) \omega^{\frac{4}{N-2}}(x) d y \\
& \leq C\left\|h_{\varepsilon}\right\|_{* *}\left(\sum_{i}\left(1+\left|x-\xi_{i}^{\prime}\right|^{2}\right)^{-\frac{N-2}{2}}\right)^{\beta} .
\end{aligned}
$$

Analogously we get

$$
\begin{aligned}
\int_{\Omega_{\varepsilon}}\left|\partial_{x_{j}} G_{\varepsilon}(x, y) h_{\varepsilon}\right| d y & \leq\left\|h_{\varepsilon}\right\|_{* *} C \sum_{j} \int_{\mathbb{R}^{N}} \frac{1}{|x-y|^{N-1}}\left(1+\left|y-\xi_{j}^{\prime}\right|^{2}\right)^{-2} d y \\
& \leq C\left\|h_{\varepsilon}\right\|_{* *} w^{\beta+\frac{1}{N-2}}(x) .
\end{aligned}
$$


On the other hand, we have

$$
\begin{aligned}
& \left|\sum c_{i} \int_{\Omega_{\varepsilon}} V^{p-1} Z_{\varepsilon i} G_{\varepsilon}(x, y) d y\right| \\
& \leq C\left(\left\|\phi_{\varepsilon}\right\|_{\rho}+\left\|h_{\varepsilon}\right\|_{* *}\right) \sum_{i} \int_{\mathbb{R}^{N}} \Gamma(x-y)\left(\left(1+\left|y-\xi_{i}^{\prime}\right|^{2}\right)^{-\frac{N+3}{2}}\right) \\
& \leq C\left(\left\|\phi_{\varepsilon}\right\|_{\rho}+\left\|h_{\varepsilon}\right\|_{* *}\right)\left(\sum_{i}\left(1+\left|x-\xi_{i}^{\prime}\right|^{2}\right)^{-\frac{N-2}{2}}\right)
\end{aligned}
$$

and

$$
\begin{gathered}
\left|\sum c_{i} \int_{\Omega_{\varepsilon}} V^{p-1} Z_{\varepsilon i} \partial_{x_{j}} G_{\varepsilon}(x, y) d y\right| \\
\leq C\left(\left\|\phi_{\varepsilon}\right\|_{\rho}+\left\|h_{\varepsilon}\right\|_{* *}\right) \sum \int_{\mathbb{R}^{N}} \frac{1}{|x-y|^{N-1}}\left(\left(1+\left|y-\xi_{i}^{\prime}\right|^{2}\right)^{-\frac{N+3}{2}}\right) \\
\leq C\left(\left\|\phi_{\varepsilon}\right\|_{\rho}+\left\|h_{\varepsilon}\right\|_{* *}\right) w^{\beta+\frac{1}{N-2}}(x) .
\end{gathered}
$$

Similarly, we obtain

$$
\int_{\Omega_{\varepsilon}}\left|G_{\varepsilon}(x, y) V^{p+\lambda-1} \phi_{\varepsilon}\right| d y \leq C\left\|\phi_{\varepsilon}\right\|_{\rho}\left(\sum_{i}\left(1+\left|x-\xi_{i}^{\prime}\right|^{2}\right)^{-\frac{N-2}{2}}\right)^{\beta}
$$

and

$$
\int_{\Omega_{\varepsilon}}\left|\partial_{x_{j}} G_{\varepsilon}(x, y) V^{p+\lambda-1} \phi_{\varepsilon}\right| d y \leq C\left\|\phi_{\varepsilon}\right\|_{\rho} w^{\beta+\frac{1}{N-2}}(x) .
$$

Equation (5.7) and (5.8) and the above estimates imply that

$$
\left|\phi_{\varepsilon}(x)\right| \leq C\left(\left\|\phi_{\varepsilon}\right\|_{\rho}+\left\|h_{\varepsilon}\right\|_{* *}\right) w^{\beta}(x)
$$

and

$$
\left|\partial_{x_{j}} \phi_{\varepsilon}(x)\right| \leq C\left(\left\|\phi_{\varepsilon}\right\|_{\rho}+\left\|h_{\varepsilon}\right\|_{* *}\right) w^{\beta+\frac{1}{N-2}}(x) .
$$

In particular, we have that

$$
w^{-(\beta-\rho)}(x)\left|\phi_{\varepsilon}(x)\right| \leq C w^{\rho}(x) .
$$

Since $\rho$ is arbitrarily small and $\left\|\phi_{\varepsilon}\right\|_{\rho}=1$, it follows the existence of a radius $R>0$ and a number $\gamma>0$, both independent of $\varepsilon$ such that $\left\|\phi_{\varepsilon}\right\|_{L^{\infty}\left(B_{R}\left(\xi^{\prime}\right)\right)}>\gamma$. Then local elliptic estimates and the bound (5.10) yield that, up to a subsequence, $\tilde{\phi}_{\varepsilon}(x)=\phi_{\varepsilon}\left(x-\xi^{\prime}\right)$ converges uniformly over compacts of $\mathbb{R}^{N}$ to a nontrivial solution $\tilde{\phi}$ of

$$
\Delta \tilde{\phi}+p U_{\Lambda, 0}^{p-1} \tilde{\phi}=0
$$


for some $\Lambda>0$, which besides satisfies

$$
|\tilde{\phi}(x)| \leq C|x|^{(2-N) \beta} .
$$

Hence, for $N=3$ we have

$$
|\tilde{\phi}(x)| \leq C|x|^{2-N} .
$$

Now, since $\tilde{\phi}$ satisfies (5.12) and estimate (5.13) holds true, a bootstrap argument leads to

$$
|\tilde{\phi}(x)| \leq C|x|^{2-N} \quad \text { for any } N>3 .
$$

It is well known that this implies that $\tilde{\phi}$ is a linear combination of the functions $\frac{\partial U_{\Lambda, 0}}{\partial x_{j}}, \frac{\partial U_{\Lambda, 0}}{\partial \Lambda}$. On the other hand, we recall that $\int_{\Omega_{\varepsilon}} \phi_{\varepsilon} V^{p-1} Z_{\varepsilon i}=0$ for all $i$. Hence passing to the limit for $\varepsilon \rightarrow 0$, we get $\int_{\mathbb{R}^{N}} \tilde{\phi} U_{\Lambda, 0}^{p-1} \frac{\partial U_{\Lambda, 0}}{\partial x_{j}}=\int_{\mathbb{R}^{N}} \tilde{\phi} U_{\Lambda, 0}^{p-1} \frac{\partial U_{\Lambda, 0}}{\partial \Lambda}=$ 0 , for all $j$. Hence the only possibility is that $\tilde{\phi} \equiv 0$, which is a contradiction which yields the proof of $\left\|\phi_{\varepsilon}\right\|_{\rho} \rightarrow 0$. Finally, from estimate (5.10), we observe that

$$
\left\|\phi_{\varepsilon}\right\|_{*} \leq C\left(\left\|h_{\varepsilon}\right\|_{* *}+\left\|\phi_{\varepsilon}\right\|_{\rho}\right),
$$

hence $\left\|\phi_{\varepsilon}\right\|_{*} \rightarrow 0$, and the proof is thus complete.

Step 2. Now we are in a position to prove Proposition 3.1. To do this, let us consider the space

$$
H=\left\{\phi \in H_{0}^{1}\left(\Omega_{\varepsilon}\right) \mid \int_{\Omega_{\varepsilon}} V^{p-1} Z_{\varepsilon i} \phi=0 \forall i\right\}
$$

endowed with the usual inner product $[\phi, \psi]=\int_{\Omega_{\varepsilon}} \nabla \phi \nabla \psi$. Denote with $\langle f, g\rangle$ the inner product in $L 2\left(\Omega_{\varepsilon}\right)$. Problem (3.8) expressed in weak form is equivalent to that of finding a $\phi \in H$ such that

$$
[\phi, \psi]=\left\langle\left((p+\lambda) V^{p+\lambda-1} \phi-h\right), \psi\right\rangle \quad \forall \psi \quad \in H .
$$

With the aid of Riesz's representation theorem, this equation gets rewritten in $H$ in the operational form $\phi=K(\phi)+\tilde{h}$ with certain $\tilde{h} \in H$ which depends linearly in $h$ and where $K$ is a compact operator in $H$. Fredholm's alternative guarantees unique solvability of this problem for any $h$ provided that the homogeneous equation $\phi=$ $K(\phi)$ has only the zero solution in $H$. Let us observe that this last equation is equivalent to (3.8). Assume it has a nontrivial solution $\phi=\phi_{\varepsilon}$, which with no loss of generality may be taken so that $\left\|\phi_{\varepsilon}\right\|_{*}=1$. But this makes the previous step applicable, so that necessarily $\left\|\phi_{\varepsilon}\right\|_{*} \rightarrow 0$. This is certainly a contradiction that proves that this equation only has the trivial solution in $H$. We conclude then that for each $h$, problem (3.8) admits a unique solution. We check that

$$
\|\phi\|_{*} \leq C\|h\|_{* *} .
$$

We assume again the opposite. In doing so, we find a sequence $h_{\varepsilon}$ with $\left\|h_{\varepsilon}\right\|_{* *}=$ $o$ (1) and solutions $\phi_{\varepsilon} \in H$ of problem (3.8) with $\left\|\phi_{\varepsilon}\right\|_{*}=1$. Again this makes the 
previous step applicable, and a contradiction has been found. This proves the first estimate in (3.9). Estimate (3.10) follows from this and relation (5.6).

Let us now consider differentiation with respect to the variable $\xi_{l}^{\prime}, l=1, \ldots, N$. For notational simplicity we write $\frac{\partial}{\partial \xi_{l}^{\prime}}=\partial_{\xi^{\prime}}$. Let us set, $\phi=T(h)$ and, still formally, $Z=\partial_{\xi^{\prime}} \phi$. We seek for an expression for $Z$. Then $Z$ satisfies the following equation

$$
\begin{aligned}
\Delta Z+(p+\lambda) V^{p+\lambda-1} Z= & -(p+\lambda) \partial_{\xi^{\prime}}\left(V^{p-1+\lambda}\right) \phi \\
& +\sum_{i} d_{i} V^{p-1} Z_{\varepsilon i}+c_{i} \partial_{\xi^{\prime}}\left(V^{p-1} Z_{\varepsilon i}\right) \quad \text { in } \Omega_{\varepsilon} .
\end{aligned}
$$

Here $d_{i}=\partial_{\xi^{\prime}} c_{i}$. Besides, from differentiating the orthogonality condition $\left\langle\phi, V^{p-1} Z_{\varepsilon i}\right\rangle=0$ we further obtain the relations

$$
\left\langle\phi, \partial_{\xi^{\prime}}\left(V^{p-1} Z_{\varepsilon i}\right)\right\rangle+\left\langle Z, V^{p-1} Z_{\varepsilon i}\right\rangle=0 .
$$

Let us consider constants $b_{j}$ such that

$$
\left\langle Z-\sum_{l} b_{l} Z_{\varepsilon l}, V^{p-1} Z_{\varepsilon i}\right\rangle=0 .
$$

These relations amount to

$$
\sum_{l} b_{l}\left\langle Z_{\varepsilon l}, V^{p-1} Z_{\varepsilon i}\right\rangle=\left\langle\phi, \partial_{\xi^{\prime}} V^{p-1} Z_{\varepsilon i}\right\rangle
$$

Since this system is diagonal dominant with uniformly bounded coefficients, we see that it is uniquely solvable and that

$$
b_{l}=O\left(\|\phi\|_{*}\right) .
$$

Now, we easily see that

$$
\left\|\phi \partial_{\xi^{\prime}}\left(V^{p-1+\lambda}\right)\right\|_{* *} \leq C\|\phi\|_{*} .
$$

Recall now that $c_{i}=O\left(\|h\|_{* *}\right)$. On the other hand

$$
\left|\partial_{\xi^{\prime}}\left(V^{p-1} Z_{\varepsilon i}(x)\right)\right| \leq C\left|x-\xi_{i}^{\prime}\right|^{-N-4},
$$

hence

$$
\left\|c_{i} \partial_{\xi^{\prime}} V^{p-1} Z_{\varepsilon i}\right\|_{* *} \leq C\|h\|_{* *} .
$$

Let us now set $\eta=Z-\sum_{j} b_{j} Z_{\varepsilon j}$. Then, summing up the estimates above, and using that $\|\phi\|_{*} \leq C\|h\|_{*_{*}}$, we get that $\eta$ satisfies the relation

$$
\Delta \eta+(p+\lambda) V^{p-1+\lambda} \eta=f+\sum_{i} d_{i} V^{p-1} Z_{\varepsilon i} \quad \text { in } \Omega_{\varepsilon},
$$


where

$$
\begin{aligned}
f= & \sum_{i} b_{i}\left(-\left(\Delta+(p+\varepsilon) V^{p-1+\lambda}\right) Z_{\varepsilon i}+c_{i} \partial_{\xi^{\prime}}\left(V^{p-1} Z_{\varepsilon i}\right)\right. \\
& -(p+\lambda) \partial_{\xi^{\prime}}\left(V^{p-1+\lambda}\right) \phi
\end{aligned}
$$

so that

$$
\|f\|_{* *} \leq C\|h\|_{* *} .
$$

Since besides $\eta \in H_{0} 1\left(\Omega_{\varepsilon}\right)$ and

$$
\left\langle\eta, V^{p-1} Z_{\varepsilon i}\right\rangle=0 \quad \text { for all } i,
$$

we have that $\eta=T(f)$. Reciprocally, if we now define

$$
Z=T(f)+\sum_{i} b_{i} Z_{\varepsilon i}
$$

with $b_{i}$ given by relations (5.14) and $f$ by (5.16), then it is a matter of routine to check that indeed $Z=\partial_{\xi^{\prime}} \phi$. In fact $Z$ depends continuously on the parameters $\xi^{\prime}$, $\Lambda$ and $h$ for the norm \|\|$_{*}$, and $\|Z\|_{*} \leq C\|h\|_{* *}$ for points in the considered region. The corresponding result for differentiation with respect to the variable $d$ follow similarly. This concludes the proof.

\section{The nonlinear problem: proof of Proposition 3.2}

Let us rewrite the first equation in (3.11) in the following form

$$
\Delta \phi+(p+\lambda) V^{p+\lambda-1} \phi=-E-N(\phi)+\sum_{i} c_{i} V^{p-1} Z_{\varepsilon i} \quad \text { in } \Omega_{\varepsilon},
$$

where $E$ and $N(\phi)$ are defined respectively by (3.6) and (3.5).

To estimate the \|\|$_{* *}$-norm of $N(\eta)$, where $\eta$ is a function with bounded $\|\cdot\|_{*^{-}}$ norm and $\eta=0$ on $\partial \Omega_{\varepsilon}$, it is convenient, and sufficient for our purposes, to assume $\|\eta\|_{*}<1$. Note that

$$
N(\eta)=N_{1}(\eta)+N_{2}(\eta)
$$

where

$$
N_{1}(\eta)=(p+\lambda)\left(1-\varepsilon^{-\frac{\lambda}{p-1}}\right) V^{p+\lambda-1} \eta
$$

and

$$
N_{2}(\eta)=\frac{(p+\lambda)(p-1+\lambda)}{2}\left(V_{1}+V_{2}+t \eta\right)^{p-2+\lambda} \eta^{2}
$$

with $t \in(0,1)$. First observe that

$$
\left|w^{-\frac{4}{N-2}} N_{1}(\eta)\right| \leq C\left(1-\varepsilon^{-\frac{\lambda}{p-1}}\right) w^{p-1+\beta}\|\eta\|_{*} \leq C|\lambda \log \varepsilon|\|\eta\|_{*} .
$$


In order to estimate $\left\|N_{2}(\eta)\right\|_{* *}$ we need to distinguish two cases: $N \leq 6$ and $N>6$. If $N \leq 6$, then $p \geq 2$ and we can estimate

$$
\left|w^{-\frac{4}{N-2}} N_{2}(\eta)\right| \leq C w^{p-2+2 \beta-\frac{4}{N-2}}\|\eta\|_{*}^{2},
$$

hence

$$
\left\|N_{2}(\eta)\right\|_{* *} \leq C\|\eta\|_{*}^{2}
$$

Assume now that $N>6$.

If $|\eta| \geq \frac{1}{2} V$, we see directly from (6.3) that $\left|N_{2}(\eta)\right| \leq C|\eta|^{p}$ and hence

$$
\left|w^{-\frac{4}{N-2}} N_{2}(\eta)\right| \leq C w^{p-2}\|\eta\|_{*}^{p} \leq C \varepsilon^{-\frac{N-6}{2}}\|\eta\|_{*}^{p} .
$$

Let us consider now the case $|\eta| \leq \frac{1}{2} V$.

In the region where $\operatorname{dist}\left(y, \partial \Omega_{\varepsilon}\right) \geq \delta \varepsilon^{-\frac{1}{2}}$, for some $\delta>0$, then $V(y) \geq$ $\alpha_{\delta} w(y)$ for some $\alpha_{\delta}>0$; hence in this region, we have

$$
\left|w^{-\frac{4}{N-2}} N_{2}(\eta)\right| \leq C w^{2 \beta-1}\|\eta\|_{*}^{2} \leq C \varepsilon^{(2 \beta-1) \frac{N-2}{2}}\|\eta\|_{*}^{2} .
$$

On the other hand, when $\operatorname{dist}\left(y, \partial \Omega_{\varepsilon}\right) \leq \delta \varepsilon^{-\frac{1}{2}}$, the following facts occur: $w(y)$, $V(y)=O\left(\varepsilon^{\frac{N-2}{2}}\right)$ and, as $y \rightarrow \partial \Omega_{\varepsilon}, V(y)=C \varepsilon^{\frac{N-1}{2}} \operatorname{dist}\left(y, \partial \Omega_{\varepsilon}\right)(1+o(1))$. This second assertion is a consequence of the fact that the Green function of the domain $\Omega$ vanishes linearly with respect to $\operatorname{dist}(x, \partial \Omega)$ as $x \rightarrow \partial \Omega$. These two facts imply that, if $\operatorname{dist}\left(y, \partial \Omega_{\varepsilon}\right) \leq \delta \varepsilon^{-\frac{1}{2}}$ and $\eta(y) \neq 0$ (otherwise $N_{2}(\eta)(y)=0$ ), then

$$
\begin{aligned}
\left|w^{-\frac{4}{N-2}} N_{2}(\eta)\right| & \leq w^{-\frac{4}{N-2}} V^{p-2}|\eta|^{2} \\
& \leq C w^{-\frac{4}{N-2}}\left(\varepsilon^{\frac{N-1}{2}} \operatorname{dist}\left(y, \partial \Omega_{\varepsilon}\right)\right)^{p-2} \operatorname{dist}\left(y, \partial \Omega_{\varepsilon}\right)^{2}|D \eta(\bar{y})|^{2} \\
& \leq C w^{-\frac{4}{N-2}+2 \beta+\frac{2}{N-2}} \varepsilon^{\frac{N-1}{2}(p-2)-\frac{p}{2}}\|\eta\|_{*}^{2} \leq C \varepsilon^{-\frac{N-6}{2}}\|\eta\|_{*}^{2} .
\end{aligned}
$$

Combining these relations we get

$$
\|N(\eta)\|_{* *} \leq \begin{cases}C\|\eta\|_{*}^{2}+|\lambda \log \varepsilon|\|\eta\|_{*} & \text { if } N \leq 6 \\ C\left(\varepsilon^{-\frac{N-6}{2}}\|\eta\|_{*}^{2}+\varepsilon^{p-2}\|\eta\|_{*}^{p}+|\lambda \log \varepsilon|\|\eta\|_{*}\right) & \text { if } N>6\end{cases}
$$

Next we estimate the term $E$. We have

$$
\begin{aligned}
|E| & \leq C\left(\left(\varepsilon^{-\frac{\lambda}{p-1}}-1\right) V^{p}+\left(V^{\lambda}-1\right) V^{p}+\left|V^{p}-U_{d, \xi^{\prime}}^{p}\right|+o\left(\varepsilon^{\frac{N-2}{2}}\right)\right) \\
& \leq C\left(|\lambda \log \varepsilon| w^{p}+|\lambda| w^{p}|\log w|+\left|V^{p}-U_{d, \xi^{\prime}}^{p}\right|+o\left(\varepsilon^{\frac{N+2}{2}}\right)\right) .
\end{aligned}
$$

Taking into account that $\left\|V^{p}-U_{d, \xi^{\prime}}^{p}\right\|_{* *} \leq C \varepsilon^{\frac{N-2}{2}}$, we get

$$
\|E\|_{* *} \leq C\left(|\lambda \log \varepsilon|+\varepsilon^{\frac{N-2}{2}}\right) .
$$


Now, we are in position to prove that problem (3.11) has a unique solution $\phi=\widetilde{\phi}+\widetilde{\psi}$, with $\widetilde{\psi}:=-T(R)$, with the required properties.

In fact, problem (3.11) is equivalent to solving a fixed point problem. Indeed $\phi=\tilde{\phi}+\tilde{\psi}$ is a solution of (3.11) if and only if

$$
\tilde{\phi}=-T(N(\tilde{\phi}+\tilde{\psi})) \equiv A(\tilde{\phi})
$$

taking into account that $\tilde{\psi}=-T(R)$.

Then we need to prove that the operator $A$ defined above is a contraction inside a properly chosen region.

First observe that, from the definition of $\tilde{\psi}$, from (6.5) and from Proposition 3.1 , we infer that

$$
\|\tilde{\psi}\|_{* *} \leq C\left(|\lambda \log \varepsilon|+\varepsilon^{\frac{N-2}{2}}\right)
$$

and, by (6.4) and the choice (2.2), for $\|\eta\|_{*} \leq 1$,

$$
\|N(\tilde{\psi}+\eta)\|_{* *} \leq\left\{\begin{array}{cl}
C\left(\|\eta\|_{*}^{2}+|\lambda \log \varepsilon|\|\eta\|_{*}+|\lambda \log \varepsilon|^{2}+\varepsilon^{N-2}\right) & \text { if } N \leq 6 \\
C\left(\varepsilon^{-\frac{N-6}{2}}\|\eta\|_{*}^{2}+\varepsilon^{p-2}\|\eta\|_{*}^{p}+|\lambda \log \varepsilon|\|\eta\|_{*}\right. & \\
\left.+\varepsilon^{-\frac{N-4}{2}}|\lambda|^{2}|\log \varepsilon|^{2}+\varepsilon^{\frac{N}{2}}\right) & \text { if } N>6
\end{array}\right.
$$

Let us set

$$
\mathcal{F}=\left\{\eta \in H_{0} 1:\|\eta\|_{*} \leq\left(|\lambda \log \varepsilon|+\varepsilon^{\frac{N-2}{2}}\right)\right\} .
$$

From Proposition 3.1, (6.6) and (2.2) we conclude that, for $\varepsilon$ sufficiently small and any $\eta \in \mathcal{F}_{r}$ we have

$$
\|A(\eta)\|_{*} \leq\left(|\lambda \log \varepsilon|+\varepsilon^{\frac{N-2}{2}}\right) .
$$

Now we will show that the map $A$ is a contraction, for any $\varepsilon$ small enough. That will imply that $A$ has a unique fixed point in $\mathcal{F}$ and hence problem (3.11) has a unique solution.

For any $\eta_{1}, \eta_{2}$ in $\mathcal{F}_{r}$ we have

$$
\left\|A\left(\eta_{1}\right)-A\left(\eta_{2}\right)\right\|_{*} \leq C\left\|N\left(\tilde{\psi}+\eta_{1}\right)-N\left(\tilde{\psi}+\eta_{2}\right)\right\|_{* *},
$$

hence we just need to check that $N$ is a contraction in its corresponding norms. By definition of $N$

$$
D_{\bar{\eta}} N(\bar{\eta})=(p+\lambda)\left[(V+\bar{\eta})_{+}^{p+\lambda-1}-V^{p+\lambda-1}\right] .
$$

Hence we get

$$
\left|N\left(\tilde{\psi}+\eta_{1}\right)-N\left(\tilde{\psi}+\eta_{2}\right)\right| \leq C \bar{V}^{p-2}|\bar{\eta}|\left|\eta_{1}-\eta_{2}\right| .
$$


for some $\bar{\eta}$ in the segment joining $\tilde{\psi}+\eta_{1}$ and $\tilde{\psi}+\eta_{2}$. Hence, we get for small enough $\|\bar{\eta}\|_{*}$,

$$
\omega^{-\frac{4}{N-2}}\left|N\left(\tilde{\psi}+\eta_{1}\right)-N\left(\tilde{\psi}+\eta_{2}\right)\right| \leq C \varepsilon^{p-2+2 \beta}\|\bar{\eta}\|_{*}\left\|\eta_{1}-\eta_{2}\right\|_{*} .
$$

We conclude that there exists $c \in(0,1)$ such that

$$
\left\|N\left(\tilde{\psi}+\eta_{1}\right)-N\left(\tilde{\psi}+\eta_{2}\right)\right\|_{* *} \leq c\left\|\eta_{1}-\eta_{2}\right\|_{*} .
$$

Arguing like in [12], we obtain the estimate (3.13).

\section{References}

[1] T. Aubin, Problemes isoperimetriques et espaces de Sobolev, J. Differential Geom. 11 (1976), 573-598.

[2] A. BAhri, "Critical Points at Infinity in Some Variational Problems", Pitman Research Notes in Mathematics Series, Vol. 182, 1989, Longman.

[3] A. BAHRI and J.M. CoRON, On a nonlinear elliptic equation involving the critical Sobolev exponent: the effect of the topology of the domain, Comm. Pure Appl. Math. 41 (1988), 253-294.

[4] A. BAHRI and Y.LI, O. REY, On a variational problem with lack of compactness: the topological effect of the critical points at infinity, Calc. Var. Partial Differential Equations 3 (1995), 67-93.

[5] M. Ben Ayed, K. El Mehdi, M. Grossi and O. Rey, A nonexistence result of single peaked solutions to a supercritical nonlinear problem, Commun. Contemp. Math. 5 (2003), 179-195.

[6] G. Bianchi and H. Egnell, A note on the Sobolev inequality, J. Funct. Anal. 100 (1991), $18-24$.

[7] L. Caffarelli, B. Gidas and J. Spruck, Asymptotic symmetry and local behaviour of semilinear elliptic equations with critical Sobolev growth, Comm. Pure Appl. Math. 42 (1989), 271-297.

[8] J. M. Coron, Topologie et cas limite des injections de Sobolev, C. R. Acad. Sci. Paris Sér. I Math. 299 (1984), 209-212.

[9] E. N. DANCER, A note on an equation with critical exponent, Bull. London Math. Soc. 20 (1988), 600-602.

[10] E. N. DANCER, Domain variation for certain sets of solutions and applications, Topol. Methods Nonlin. Anal. 7 (1996), 95-113.

[11] E. N. DANCER, Superlinear problems on domains with holes of asymptotic shape and exterior problems, Math. Z. 229 (1998), 475-491.

[12] M. Del Pino, P. Felmer and M. Musso, Two-bubble solutions in the super-critical Bahri-Coron's problem, Calc. Var. Partial Differential Equations 16 (2003), 113-145.

[13] M. Del Pino, P. FElmer and M. Musso, Multi-peak solutions for super-critical elliptic problems in domains with small holes, J. Differential Equations 182 (2002), 511-540.

[14] M. DEL Pino, P. Felmer and M. Musso, Multi-bubble solutions for Slightly super-critial ellitpic problems in domains with symmetries, Bull. London Math. Soc. 35 (2003), 513-521.

[15] Z. C. HAN, Asymptotic approach to singular solutions for nonlinear elliptic equations involving critical Sobolev exponent, Ann. Inst. H. Poincaré Anal. Non Linéaire 8 (1991), $159-174$.

[16] J. KAZDAN and F. W. WARNER, Remarks on some quasilinear elliptic equations, Comm. Pure Appl. Math. 28 (1975), 567-597. 
[17] S. KHENISSY and O. REY, A criterion for existence of solutions to the supercritical BahriCoron's problem, Houston J. Math. 30 (2004), 587-613.

[18] R. LEWANDOWSKI, Little holes and convergence of solutions of $-\Delta u=u^{(N+2) /(N-2)}$, Nonlinear Anal. 14 (1990), 873-888.

[19] G. LI, S. YAN and J. YANG, An elliptic problem with critical growth in domains with shrinking holes, J. Differential Equations 198 (2004), 275-300.

[20] R. Molle and D. PASsaseo, Positive solutions for slightly super-critical elliptic equations in contractible domains, C. R. Math. Acad. Sci. Paris 335 (2002), 459-462.

[21] A. PistoiA and O. REY, Multiplicity of solutions to the supercritical Bahri-Coron's problem in pierced domains, Adv. Differential Equations 11 (2006), 647-666.

[22] S. I. PoHožAEV, On the eigenfunctions of the equation $\Delta u+\lambda f(u)=0$, (Russian) Dokl. Akad. Nauk 165 (1965), 36-39.

[23] O. ReY, Proof of two conjectures of H. Brezis and L.A. Peletier, Manuscripta Math. 65 (1989), 19-37.

[24] O. REY, Sur un probléme variationnel non compact: l'effect de petits trous dans le domain, C.R. Acad. Sci. Paris 308 (1989), 349-352.

[25] O. REY, Blow-up points of solutions to elliptic equations with limiting nonlinearity, Differential Integral Equations 4 (1991), 1155-1167.

[26] G. TAlenti, Best constants in Sobolev inequality, Ann. Mat. Pura Appl. 110 (1976), 353372.

Departamento de Matematica

Pontificia Universidad Catolica de Chile

Avenida Vicuna Mackenna 4860

Macul, Santiago, Chile

and

Dipartimento di Matematica

Politecnico di Torino

Corso Duca degli Abruzzi, 24

10129 Torino, Italy

mmusso@mat.puc.cl.

Dipartimento di Metodi

e Modelli Matematici

Sapienza Università di Roma

via A. Scarpa 16

00161 Roma, Italy

pistoia@dmmm.uniroma1.it. 